\title{
A local limit theorem for perturbed random walks
}

\author{
Mei Wang \\ Department of Mathematics, The University of Michigan, Ann Arbor, MI, USA
}

Received August 1990

Abstract: The main result reported here is a Stone type local limit theorem for perturbed random walks $Z_{n}=S_{n}+\xi_{n}$ when some slow variation conditions are imposed on $\xi_{n}$ 's.

Keywords: Local (central) limit theorem; Edgeworth expansion; perturbed random walks.

\section{Background}

When specialized to one dimension and non-lattice distributions, Stone's Theorem (1965) asserts the following: Let $X_{1}, X_{2}, X_{3}, \ldots$ denote i.i.d. non-lattice random variables for which $\mathbb{E}\left[X_{i}\right]=0$ and $\mathbb{E}\left[X_{i}^{2}\right]=1$. Let

$$
S_{n}=X_{1}+X_{2}+\cdots+X_{n}, \quad n=1,2,3, \ldots,
$$

be the sums, called 'random walks'. Then for each $L \in[0, \infty)$,

$$
\varepsilon_{n}(L)=\sup _{c \leqslant L} \sup _{b \in \mathbb{B}}\left|\sqrt{n} \mathbb{P}\left\{b<S_{n} \leqslant b+c\right\}-c \phi(b / \sqrt{n})\right| \underset{n \rightarrow \infty}{\longrightarrow} 0,
$$

where $\phi(x)$ is the standard normal density and $\mathbb{R}$ is the real line. A consequence of this is

$$
\sqrt{n} \mathbb{P}\left\{S_{n} \in J\right\} \underset{n \rightarrow \infty}{\longrightarrow}|J| / \sqrt{2 \pi}
$$

for any interval $J \subset \mathbb{R}$ of length $|J|$. This is the result of Shepp (1964).

There has been recent interest in sequences of random variables, called perturbed random walks. See, for example, Siegmund (1985), Woodroofe (1982) and Lalley (1984). A perturbed random walk has the form

$$
Z_{n}=S_{n}+\xi_{n}
$$

where $S_{n}$ is a random walk, $\xi_{n}$ is independent of $X_{n+1}, X_{n+2}, \ldots$ for all $n=1,2, \ldots$ and the sequence $\xi_{n}, n \geqslant 1$, is slowly changing in a sense described by Woodroofe $(1982$, p. 41$)$.

Correspondence to: Prof. Mei Wang, Department of Mathematics, University of Michigan, Ann Arbor, MI 48109-1003, USA.

Research partially supported by the U.S. Army Research Office under DAAL 03-88-K-0122. 


\section{A local limit theorem}

The main result of this paper is a local limit theorem for perturbed random walks. The following conditions are imposed.

Condition C (Cramer's condition). A random variable $X_{i}$ is said to satisfy Condition $\mathrm{C}$ if the characteristic function

$$
\psi(t)=\mathbb{E}\left[\mathrm{e}^{\mathrm{i} t X_{i}}\right]=\int_{\mathbb{R}} \mathrm{e}^{\mathrm{i} t x} F(\mathrm{~d} x), \quad t \in \mathbb{R},
$$

has the property

$$
\limsup _{t \rightarrow \infty}|\psi(t)|<1
$$

Cramer's condition is also known as the strongly non-lattice condition.

Condition SL (Slow variation condition on $\xi_{i}$ 's). A sequence of random variables, $\xi_{i}$, is said to satisfy Condition SL if there is a number $\alpha \in\left(\frac{1}{2}, 1\right)$, such that

$$
\lim _{n \rightarrow \infty} \sqrt{n} \mathbb{P}\left\{\left|\xi_{n}-\xi_{n-\left[n^{\alpha}\right]}\right| \geqslant \varepsilon\right\}=0
$$

for each $\varepsilon>0$.

Condition $\mathbf{M}$ (Moment condition on $\xi_{i}$ 's). A sequence of random variables, $\xi_{i}$, is said to satisfy Condition $\mathrm{M}$ if for the $\alpha$ in Condition SL,

$$
\lim _{n \rightarrow \infty} \frac{\mathbb{E}\left[\left|\xi_{n}\right|\right]}{n^{\alpha-1 / 2}}=0 \text {. }
$$

For $\xi_{n}$ satisfying Condition SL and M, and independent of $X_{n+1}, X_{n+2}, \ldots$ for all $n=1,2, \ldots$, the sequence $Z_{n}=S_{n}+\xi_{n}$ is called a perturbed random walk, even though the conditions imposed differ from those imposed for nonlinear renewal theory.

Theorem 1 (Stone type local limit theorem). If $\mathbb{E}\left|X_{i}^{3}\right|<\infty$ and Conditions $\mathrm{C}$, SL and $\mathrm{M}$ hold, then

$$
\varepsilon_{n}(L)=\sup _{0 \leqslant c \leqslant L} \sup _{b \in \mathbb{R}}\left|\sqrt{n} \mathbb{P}\left\{b<Z_{n} \leqslant b+c\right\}-c \phi(b / \sqrt{n})\right| \underset{n \rightarrow \infty}{\longrightarrow} 0
$$

for any $L<\infty$.

Examples. A class of examples of perturbed random walks may be constructed by letting

$$
Z_{n}=n g\left(S_{n} / n\right)
$$

where $g(0)-0, g^{\prime}(0)=1$, and $g \in \mathscr{E}^{2}([-\delta, \delta]$ ) (i.e., $g$ is twice continuously differentiablc on $[-\delta, \delta]$ ) for some $\delta>0$. Assume $\mathbb{E}\left|X_{i}^{3}\right|<\infty$ and Condition $C$ holds for the $X_{i}$ 's. Write

$$
\xi_{n}=n g\left(S_{n} / n\right)-S_{n}=Z_{n}-S_{n}, \quad n \in \mathbb{N} .
$$

If there is a $k \geqslant 0$ and $C>0$ such that

$$
|g(x)| \leqslant C(|x|+1)^{k}, \quad x \in \mathbb{R}
$$


and $\mathbb{E}\left|X_{i}^{p}\right|<\infty$ for some $p>k$, then $Z_{n}=S_{n}+\xi_{n}$ satisfies all the conditions in the local limit theorem for perturbed random walks (Theorem 1 ).

The verification of these assertions will be given in the last section.

The following lemma is an important tool in the proof of the theorem.

Lemma 2 (Edgeworth expansion, Woodroofe, 1988). Assume $\mathbb{E}\left|X_{i}^{3}\right|<\infty$ and Condition $\mathrm{C}$ holds. Let $M$ be given, $0<M<\infty$. Then for $b \in \mathbb{R}$ and $c \in[0, M]$,

$$
F^{* n}(b+c)-F^{* n}(b-c)=\Phi((b+c) / \sqrt{n})-\Phi((b-c) / \sqrt{n})+\mathrm{O}(1 / n)
$$

uniformly in $b$ and $c$.

The lemma follows directly from Theorem 1 in Section 3 of Woodroofe (1988).

Proof of Theorem 1. For $0 \leqslant \beta<\gamma<\infty$ and $n \geqslant 1$, let

$$
\varepsilon_{n}(\beta, \gamma)=\sup _{\beta \leqslant c \leqslant \gamma} \sup _{b \in \mathbb{B}}\left|\sqrt{n} \mathbb{P}\left\{b<Z_{n} \leqslant b+c\right\}-c \phi(b / \sqrt{n})\right| .
$$

Then it is easily seen that

$$
\varepsilon_{n}(L)=\varepsilon_{n}(0, L) \leqslant \varepsilon_{n}(0, \delta)+\varepsilon_{n}(\delta, L) \quad \forall \delta \in(0, L) .
$$

So it is sufficient to show that for all $\delta>0, \varepsilon_{n}(\delta, L) \rightarrow 0$ as $n \rightarrow \infty$.

Let $\alpha \in\left(\frac{1}{2}, 1\right)$ be as in Condition SL. Let $b \in \mathbb{R}, c \in[\delta, L]$ and $\varepsilon \in\left(0, \frac{1}{2} \delta\right)$. For sufficiently large $n$, let $l=\left[n^{\alpha}\right]$ and $m=n-l$. Then

$$
\begin{aligned}
\mathbb{P}\left\{b<Z_{n} \leqslant b+c\right\} & \leqslant \mathbb{P}\left\{b<Z_{n} \leqslant b+c,\left|\xi_{n}-\xi_{m}\right|<\varepsilon\right\}+\mathbb{P}\left\{\left|\xi_{n}-\xi_{m}\right| \geqslant \varepsilon\right\} \\
& \leqslant \mathbb{P}\left\{b-\varepsilon-Z_{m}<S_{n}-S_{m} \leqslant b+\varepsilon-Z_{m}+c\right\}+\delta_{n} / \sqrt{n}
\end{aligned}
$$

where $\delta_{n} \rightarrow 0$ as $n \rightarrow \infty$ by Condition SL. Let $\mathscr{B}_{m}=\sigma\left\{X_{1}, X_{2}, \ldots, X_{m} ; \xi_{1}, \xi_{2}, \ldots, \xi_{m}\right\}$ be the $\sigma$-field generated by $\left\{X_{1}, X_{2}, \ldots, X_{m}, \xi_{1}, \xi_{2}, \ldots, \xi_{m}\right\}$ and

$$
\Psi_{n}(x, c)=F^{* l}(x+c+\varepsilon)-F^{* l}(x-\varepsilon) .
$$

Then

$$
\begin{aligned}
& \mathbb{P}\left\{b-\varepsilon-Z_{m}<S_{n}-S_{m} \leqslant b+\varepsilon-Z_{m}+c\right\} \\
& \quad= \mathbb{E}\left[\mathbb{P}\left\{b-\varepsilon-Z_{m}<S_{n}-S_{m} \leqslant b+\varepsilon-Z_{m}+c \mid \mathscr{B}_{m}\right\}\right] \\
& \quad=\mathbb{E}\left[\Psi_{n}\left(b-Z_{m}, c\right)\right] .
\end{aligned}
$$

Similarly,

$$
\mathbb{P}\left\{b-\varepsilon<S_{n} \leqslant b+c+\varepsilon\right\}=\mathbb{E}\left[\Psi_{n}\left(b-S_{m}, c\right)\right] .
$$

So

$$
\begin{aligned}
\sqrt{n} \mathbb{P}\left\{b<Z_{n} \leqslant b+c\right\} \leqslant & \sqrt{n} \mathbb{P}\left\{b-\varepsilon<S_{n} \leqslant b+\varepsilon+c\right\} \\
& +\sqrt{n} \mathbb{E}\left[\left|\Psi_{n}\left(b-Z_{m}, c\right)-\Psi_{n}\left(b-S_{m}, c\right)\right|\right]+\delta_{n} .
\end{aligned}
$$

The first term on the right-hand side will give the desired upper bound by Stone's theorem. The second term will approach zero as $n \rightarrow \infty$ by using the local Edgeworth expansion (Lemma 2). The third term goes to zero as $n \rightarrow \infty$ as above. 
For the first term, by Stone's theorem,

$$
\left|\sqrt{ } n \mathbb{P}\left\{b-\varepsilon<S_{n} \leqslant b+\varepsilon+c\right\}-(c+2 \varepsilon) \phi((b-\varepsilon) / \sqrt{ } n)\right| \underset{n \rightarrow \infty}{\longrightarrow} 0
$$

uniformly for $b \in \mathbb{R}$ and $c \in[\delta, L]$.

By Lemma 2 and a Taylor series expansion, there are constants $C_{i}(L)$ depending only on $L$ for which

$$
\begin{aligned}
& \sqrt{n}\left|\Psi_{n}\left(b-Z_{m}, c\right)-\Psi_{n}\left(b-S_{m}, c\right)\right| \\
& \leqslant \sqrt{n} \mid \Phi\left(\frac{b+c+\varepsilon-Z_{m}}{\sqrt{l}}\right)-\Phi\left(\frac{b-\varepsilon-Z_{m}}{\sqrt{l}}\right) \\
& \quad-\left[\Phi\left(\frac{b+c+\varepsilon-S_{m}}{\sqrt{l}}\right)-\Phi\left(\frac{b-\varepsilon-S_{m}}{\sqrt{l}}\right)\right] \mid+C_{1}(L) \frac{\sqrt{n}}{l} \\
& \leqslant \sqrt{n}\left|\left[\phi\left(\frac{b-\varepsilon-Z_{m}}{\sqrt{l}}\right)-\phi\left(\frac{b-\varepsilon-S_{m}}{\sqrt{l}}\right)\right] \frac{c+2 \varepsilon}{\sqrt{l}}\right|+\frac{\sqrt{n}}{l}\left[\left(\max \phi^{\prime}\right)(c+2 \varepsilon)^{2}+C_{1}(L)\right] \\
& \leqslant C_{2}(L) \frac{\sqrt{n}}{l}\left|Z_{m}-S_{m}\right|+C_{3}(L) \frac{\sqrt{n}}{l}
\end{aligned}
$$

for all $\mathrm{b} \in \mathbb{R}$ and $c \leqslant L$. Observe that the last line does not depend on $b$ or $c$. For large $n, l \geqslant \frac{1}{2} n^{\alpha}$. So by Condition $\mathrm{M}$,

$$
\mathbb{E}\left[\frac{\sqrt{n}}{l}\left|Z_{m}-S_{m}\right|\right] \leqslant 2 \frac{\mathbb{E}\left[\left|\xi_{m}\right|\right]}{n^{\alpha-1 / 2}} \underset{n \rightarrow \infty}{\longrightarrow} 0 .
$$

It follows from (1) and (2) that there is a sequence $\sigma_{n}, n \geqslant 1$, for which

$$
\sqrt{n} \mathbb{P}\left\{b<Z_{n} \leqslant b+c\right\}-(c+2 \varepsilon) \phi((b-\varepsilon) / \sqrt{n}) \leqslant \sigma_{n} \underset{n \rightarrow \infty}{\longrightarrow} 0,
$$

uniformly in $\mathrm{b} \in \mathbb{R}, c \in[\delta, L]$. Similarly, there is a sequence $\hat{\sigma}_{n}$ with $\hat{\sigma}_{n} \rightarrow 0$ as $n \rightarrow \infty$ and

$$
\sqrt{n} \mathbb{P}\left\{b-Z_{n} \leqslant b+c\right\}-(c-2 \varepsilon) \phi((b+\varepsilon) / \sqrt{n}) \geqslant \hat{\sigma}_{n} \underset{n \rightarrow \infty}{\longrightarrow} 0
$$

uniformly in $b \in \mathbb{R}, c \in[\delta, L]$.

The theorem follows by letting $n \rightarrow \infty$ and then $\varepsilon \downarrow 0$.

\section{Directly Riemann integrable functions}

The following definition is taken from Feller (1966). For a bounded real valued function $h$ defined on $\mathbb{R}$, $\delta>0$ and $k \in \mathbb{Z}$, let $J_{k}=(k \delta,(k+1) \delta]$ and

$$
\begin{array}{ll}
m_{k}=\min \left\{h(x): x \in J_{k}\right\}, & M_{k}=\max \left\{h(x): x \in J_{k}\right\}, \\
\bar{\sigma}=\bar{\sigma}_{\delta}(h)=\sum_{k=-\infty}^{\infty} \delta M_{k}, \quad \underline{\sigma}=\underline{\sigma}_{\delta}(h)=\sum_{k=-\infty}^{\infty} \delta m_{k} .
\end{array}
$$

Then $h$ is said to be directly Ricmann integrable $(h \in \mathrm{DR}$ ) if: (a) $\bar{\sigma}, \underline{\sigma}$ converge absolutely for sufficiently small $\delta>0$; (b) $\lim _{\delta \rightarrow 0}\left(\bar{\sigma}_{\delta}-\underline{\sigma}_{\delta}\right)=0$. Then $h \in L^{1}$ and for all $\varepsilon>0$,

$$
\underline{\sigma}_{\varepsilon} \leqslant \lim _{\delta \rightarrow 0} \bar{\sigma}_{\delta}=\int_{\mathbb{R}} h(x) \mathrm{d} x=\lim _{\delta \rightarrow 0} \underline{\sigma}_{\delta} \leqslant \bar{\sigma}_{\varepsilon} .
$$


Theorem 3. Suppose that conditions of Theorem 1 are satisfied. If $h \in \mathrm{DR}$, then

$$
\lim _{n \rightarrow \infty} \sqrt{n} \mathbb{E}\left[h\left(Z_{n}\right)\right]=\frac{1}{\sqrt{2 \pi}} \int_{\mathbb{R}} h(x) \mathrm{d} x .
$$

Proof. There is no loss of generality in supposing that $h \geqslant 0$, since otherwise positive and negative parts may be considered separately.

Given $\varepsilon>0$, there is a $\delta>0$ for which $\bar{\sigma}_{\delta}-\underline{\sigma}_{\delta} \leqslant \varepsilon$. With this choice of $\delta$, by Theorem 1 there is an $N_{0} \in \mathbb{N}$ for which

$$
\left|\sqrt{n} \mathrm{P}\left\{k \delta<Z_{k} \leqslant(k+1) \delta\right\}-\delta \phi(k \delta / \sqrt{n})\right| \leqslant \varepsilon \delta / \sqrt{2 \pi}
$$

for all $n \geqslant N_{0}$ and all $k \in \mathbb{Z}$. There is $K \in \mathbb{N}$ for which

$$
\sum_{|k|>K} m_{k}(\delta) \leqslant \sum_{|k|>K} M_{k}(\delta) \leqslant \varepsilon
$$

and there is an $N_{1} \in \mathbb{N}$ for which

$$
(1-\varepsilon) / \sqrt{2 \pi} \leqslant \phi(k \delta / \sqrt{n}) \leqslant 1 / \sqrt{2 \pi}
$$

for all $|k|<K, n \geqslant N_{1}$. Then

$$
\begin{aligned}
\sqrt{n} \mathbb{E}\left[h\left(Z_{n}\right)\right] & \leqslant \sum_{k \in \mathbb{Z}} M_{k} \sqrt{n} \mathbb{P}\left\{k \delta<Z_{n} \leqslant(k+1) \delta\right\} \\
& \leqslant \sum_{k \in \mathbb{Z}} M_{k}[\delta \phi(k \delta / \sqrt{n})+\varepsilon \delta / \sqrt{2 \pi}] \\
& \leqslant \sum_{k \in \mathbb{Z}} \delta M_{k}(1 / \sqrt{2 \pi}+\varepsilon / \sqrt{2 \pi}) \\
& \leqslant \frac{1+\varepsilon}{\sqrt{2 \pi}}\left(\int_{\mathbb{R}} h(x) \mathrm{d} x+\varepsilon\right)
\end{aligned}
$$

and for all $n \geqslant N_{0} \vee N_{1}$ (i.e., $\left.n \geqslant \max \left(N_{0}, N_{1}\right\}\right)$. Similarly,

$$
\sqrt{n} \mathbb{E}\left[h\left(Z_{n}\right)\right] \geqslant \frac{1-2 \varepsilon}{\sqrt{2 \pi}}\left[\left(\int_{a} h(x) \mathrm{d} x-\varepsilon\right)-\delta \varepsilon\right] .
$$

The theorem now follows by letting $n \rightarrow \infty, \delta \rightarrow 0$ and then $\varepsilon \rightarrow 0$.

The local limit theorem (Theorem 1) is the special case when $h(x)=1_{(b, b+c)^{*}}$

\section{Proof of the examples}

Recall the Marcinkiewicz-Zygmund Inequality (see Section 10.3 in Chow and Teicher, 1978): Assume $\left.\mathbb{E}\left|X_{i}\right|^{p}\right]<\infty$, for some $p \geqslant 3$. Then

$$
\mathbb{E}\left[\left|S_{n}\right|^{p}\right] \leqslant \mathbb{E}\left[\left|X_{i}\right|^{p}\right] C n^{p / 2}
$$

for some constant $C$ depending on $p$. 
Proposition 4. Suppose $g \in \mathscr{C}^{2}([-\delta, \delta])$ for some $\delta>0$ and there exists a constant $C>0$ such that $|g(x)| \leqslant C(1+|x|)^{k}$ for all $x \in \mathbb{R}$. If $\mathbb{E}\left[\left|X_{i}\right|^{p}\right]<\infty$ for some $p$ with $p>k$ and $p \geqslant 3$ where the $X_{i}$ 's satisfy condition $C$, then with $\xi_{n}=n g\left(S_{n} / n\right)-S_{n}, Z_{n}=S_{n}+\xi_{n}$ satisfies Theorem 1 .

Proof. Let $A_{n}=\left\{\left|\bar{X}_{n}\right| \leqslant \delta\right\}$. Then on $A_{n}, \xi_{n}=g^{\prime \prime}\left(\Delta_{n}\right) S_{n}^{2} / n,\left|\Delta_{n}\right| \leqslant \delta$ and $\left|g^{\prime \prime}\left(\Delta_{n}\right) 1_{A_{n}}\right| \leqslant C_{1}$ for some $C_{1} \in(0, \infty)$ independent of $n$. Let $h(x)=g(x)-x$. So $h \in \mathscr{C}^{2}([-\delta, \delta]), h(0)=0, h^{\prime}(0)=0, \xi_{n}=n h\left(S_{n} / n\right)$. It is casy to see that $|h(x)| \leqslant C_{2}|x|^{k}$ for all $|x| \geqslant \delta$ for some constant $C_{2} \in(0, \infty)$, and that $|h(x)| \leqslant C_{3} x^{2}$, for $x \in[-\delta, \delta]$, for some $C_{3} \in(0, \infty)$.

Step 1. Show that condition $\mathrm{M}$ is satisfied. i.e., $\mathbb{E}\left[\left|\xi_{n}\right|\right]=\mathrm{o}\left(n^{\alpha-1 / 2}\right)$ for some $\alpha \in\left(\frac{1}{2}, 1\right)$. Choose $\alpha \in\left(\frac{1}{2}, 1-1 / p\right) \subseteq\left(\frac{1}{2}, 1\right)$. Now

$$
\mathbb{E}\left[\left|\xi_{n}\right|\right] \leqslant \mathbb{L}\left[\left|\xi_{n}\right| 1_{A_{n}}\right]+\mathbb{E}\left[\left|\xi_{n}\right| 1_{A_{n}^{\prime}}\right]
$$

where $A_{n}^{\prime}$ is the complement of $A_{n}$. By the boundedness of $g^{\prime \prime}$ on $A_{n}$ and $\mathrm{M}-\mathrm{Z}$ inequality,

$$
\mathbb{E}\left[\left|\xi_{n}\right| 1_{A_{n}}\right]=\mathbb{E}\left[\left|g^{\prime \prime}\left(\Delta_{n}\right)\right| 1_{A_{n}} S_{n}^{2} / n\right] \leqslant C_{1} \mathbb{E}\left[S_{n}^{2}\right] / n \leqslant C_{2}=\mathrm{o}\left(n^{\alpha-1 / 2}\right) .
$$

By the properties of $h(x)$,

$$
\begin{aligned}
\mathbb{E}\left[\left|\xi_{n}\right| 1_{\mathcal{A}_{n}^{\prime}}\right] & =n \mathbb{E}\left[\left|h\left(S_{n} / n\right)\right| 1_{\mathcal{A}_{n}^{\prime}}\right] \leqslant n C_{3} \mathbb{E}\left[\left|S_{n} / n\right|^{k} 1_{A_{n}^{\prime}}\right] \\
& \leqslant C_{4} n^{2-p}=\mathrm{o}\left(n^{\alpha-1 / 2}\right) \quad(\text { since } p \geqslant 3) .
\end{aligned}
$$

The last line is a direct corollary of $\mathrm{M}-\mathrm{Z}$ inequality. So Condition $\mathrm{M}$ is satisfied.

Step 2. Show that Condition SL holds. i.c.,

$$
\mathbb{P}\left\{\left|\xi_{n}-\xi_{n-\left[n^{\alpha}\right]}\right|>\varepsilon\right]=\mathrm{o}\left(n^{-1 / 2}\right) \quad \forall \varepsilon>0 .
$$

Write $m=n-\left[n^{\alpha}\right]$. Then on $A_{m} \cap A_{n}$,

$$
\begin{aligned}
\xi_{n}-\xi_{m} & =n h\left(\bar{X}_{n}\right)-m h\left(\bar{X}_{m}\right) \\
& =n h^{\prime}\left(\bar{X}_{m}\right)\left(\bar{X}_{n}-\bar{X}_{m}\right)+\frac{1}{2} n h^{\prime \prime}\left(\Delta_{n, m}\right)\left(\bar{X}_{n}-\bar{X}_{m}\right)^{2}+(m-n) h\left(\bar{X}_{m}\right) \\
& =I_{1}+I_{2}+I_{3} .
\end{aligned}
$$

where $\Delta_{n, m}$ is between $\overline{\mathrm{X}}_{n}$ and $\bar{X}_{m}$ and $I_{i}$ denotes the $i$ th term. The Markov Inequality and M-Z inequality give

$$
\mathbb{P}\left\{A_{n}^{\prime} \cup A_{m}^{\prime}\right\} \leqslant \mathbb{E}\left[\left|\bar{X}_{n}\right|^{p}\right] / \delta^{p}+\mathbb{E}\left[\left|\bar{X}_{m}\right|^{p}\right] / \delta^{p} \leqslant C_{1}(p) n^{-p / 2}=\mathrm{o}\left(n^{-1 / 2}\right) .
$$

So it suffices to show that

$$
\mathbb{P}\left\{\left|I_{1}+I_{2}+I_{3}\right| 1_{A_{n} \cap A_{m}}>\varepsilon\right\}=\mathrm{o}\left(n^{-1 / 2}\right) \quad \forall \varepsilon>0 .
$$

By the Markov inequality, it is enough to prove that

$$
\mathbb{E}\left[\left|I_{i}\right| 1_{A_{n} \cap A_{m}}\right]=\mathrm{o}\left(n^{-1 / 2}\right) \quad \forall \varepsilon>0, \quad \text { for } i=1,2,3 .
$$

By the independence of the $X_{i}$ 's,

$$
\mathbb{E}\left[I_{1} \mid \mathscr{B}_{m}\right]=n h^{\prime}\left(\bar{X}_{m}\right) \mathbb{E}\left[\bar{X}_{n}-\bar{X}_{m} \mid \mathscr{B}_{m}\right]=h^{\prime}\left(\bar{X}_{m}\right)(m-n) \bar{X}_{m} .
$$


Since $h \in \mathscr{C}^{2}([-\delta, \delta])$ and $h^{\prime}(0)=0$, there is a constant $C_{1}$ for which $\left|h^{\prime}(x)\right| \leqslant C_{1}|x|$ for all $x \in$ $[-\delta, \delta]$. By $\mathrm{M}-\mathrm{Z}$ inequality,

$$
\begin{aligned}
\mathbb{E}\left[\left|\mathbb{E}\left[I_{1} \mid \mathscr{B}_{m}\right] 1_{A_{m}}\right|^{p / 2}\right] & \leqslant C_{1}^{p / 2}(n-m)^{p / 2} \mathbb{E}\left[\left|\bar{X}_{m}\right|^{p}\right] \\
& \leqslant C_{2}(p)((n-m) / m)^{p / 2} \\
& \leqslant C_{3}(p) n^{p(\alpha-1) / 2}=\mathrm{o}\left(n^{-1 / 2}\right) \quad(\text { since } \alpha<1-1 / p) .
\end{aligned}
$$

Let $S_{n, m}=X_{m+1}+X_{m+2}+\cdots+X_{n}$. Then $I_{1}-\mathbb{E}\left[I_{1} \mid \mathscr{B}_{m}\right]=h^{\prime}\left(\bar{X}_{m}\right) S_{n, m}$. Using the independence of $\bar{X}_{m}$ and $S_{n, m}$ and the property of $h(x)$ in $[-\delta, \delta], \mathrm{M}-\mathrm{Z}$ inequality gives

$$
\begin{aligned}
\mathbb{E}\left[\left|I_{1}-\mathbb{E}\left[I_{1} \mid \mathscr{B}_{m}\right] 1_{A_{m}}\right|^{p}\right] & \leqslant C^{p} \mathbb{E}\left[\left|\bar{X}_{m}\right|^{p}\right] \mathbb{E}\left[\left|S_{n, m}\right|^{p}\right] \\
& \leqslant C_{1}(p) m^{-p / 2}(n-m)^{p / 2} \\
& \leqslant C_{2}(p) n^{-p(1-\alpha) / 2}=\mathrm{o}\left(n^{-1 / 2}\right) .
\end{aligned}
$$

Consequently, for all $\varepsilon>0$,

$$
\mathbb{E}\left[\left|I_{1}\right| 1_{A_{n} \cap A_{m}}\right] \leqslant \mathbb{E}\left[\left|\mathbb{E}\left[I_{1} \mid \mathscr{B}_{m}\right] 1_{A_{m}}\right|\right]+\mathbb{E}\left[\left|I_{1}-\mathbb{E}\left[I_{1} \mid \mathscr{B}_{m}\right]\right| 1_{A_{m}}\right]=\mathrm{o}\left(n^{-1 / 2}\right)
$$

To prove $\mathbb{E}\left[\left|I_{2}\right| 1_{A_{n} \cap A_{m}}\right]=\mathrm{o}\left(n^{-1 / 2}\right)$, note that there is a constant $C \in(0, \infty)$ for which $\left|h^{\prime \prime}\left(\Delta_{n, m}\right) 1_{A_{m} \cap A_{n}}\right| \leqslant C$. Then

$$
\begin{aligned}
\left|I_{2}\right| 1_{A_{n} \cap A_{m}} & =\frac{1}{2} n\left|h^{\prime \prime}\left(\Delta_{n, m}\right)\right|\left(\bar{X}_{n}-\bar{X}_{m}\right)^{2} 1_{A_{n} \cap A_{m}} \\
& \leqslant \frac{1}{2} n C\left(\bar{X}_{n}-\bar{X}_{m}\right)^{2} \\
& =\frac{1}{2} n C\left(\frac{1}{n} S_{n, m}-\frac{n-m}{n m} S_{m}\right)^{2} \\
& \leqslant C_{1}\left(\frac{1}{n} S_{n, m}^{2}+\frac{(n-m)^{2}}{n m^{2}} S_{m}^{2}\right) .
\end{aligned}
$$

Therefore,

$$
\begin{aligned}
\mathbb{E}\left[\left|I_{2}\right|^{p / 2} 1_{A_{n} \cap A_{m}}\right] & \leqslant C_{2}\left\{\frac{1}{n^{p / 2}} \mathbb{E}\left[\left|S_{n, m}\right|^{p}\right]+\frac{(n-m)^{p}}{n^{p / 2} m^{p}} \mathbb{E}\left[\left|S_{m}\right|^{p}\right]\right\} \\
& \leqslant C_{3}\left\{\left(\frac{n-m}{n}\right)^{p / 2}+\left(\frac{n-m}{n}\right)^{p}\right\}=\mathrm{o}\left(n^{-1 / 2}\right) .
\end{aligned}
$$

Since $|h(x)| \leqslant C x^{2}$ on $[-\delta, \delta], \mathrm{M}-\mathrm{Z}$ inequality yields

$$
\mathbb{E}\left[\left|I_{3}\right|^{p / 2} 1_{A_{n} \cap A_{m}}\right] \leqslant C \mathbb{E}\left[(n-m)^{p / 2}\left|\bar{X}_{m}\right|^{p} 1_{A_{m}}\right] \leqslant C_{1}\left(\left[n^{\alpha}\right]\right)^{p / 2} m^{-p / 2}=\mathrm{o}\left(n^{-1 / 2}\right) .
$$

The proposition follows.

\section{Note}

The results presented here are part of the author's dissertation under the direction of M. Woodroofe. 


\section{References}

Chow, Y.S. and H. Teicher (1978), Probability Theory (Springer, New York).

Feller, W. (1966), An Introduction to Probability Theory and its Applications, Vol. 2 (Wiley, New York).

Lalley, S. (1984), Limit theorems for first passage times in linear and nonlinear renewal theory, Adv. Appl. Probab. 16, 766-803.

Shepp, L.A. (1964), A local limit theorem, Ann. Math. Statist. 35, 419-423.
Siegmund, D. (1985), Sequential Analysis (Springer, New York).

Stone, C. (1965), A local limit theorem for non-lattice multidimensional distribution functions, Ann. Math. Statist. 36, 546-551.

Woodroofe, M. (1982), Nonlinear Renewal Theory in Sequential Analysis (SIAM, Philadelphia, PA).

Woodroofe, M. (1988), Asymptotic expansions for first passage times, Stochastic Proccss. Appl. 28, 301315. 